Research Article

\title{
Preeclampsia and the Risk of Pancreatitis: A Nationwide, Population-Based Cohort Study
}

\author{
Jia-Lun Huang, ${ }^{1,2,3}$ Wei-Kung Chen, ${ }^{1,2}$ Cheng-Li Lin, ${ }^{4,5}$ Chia-Hung Kao, ${ }^{6,7}$ \\ and Hong-Mo Shih $\mathbb{1}^{1,2,3}$ \\ ${ }^{1}$ School of Medicine, College of Medicine, China Medical University, Taiwan \\ ${ }^{2}$ Department of Emergency Medicine, China Medical University Hospital, Taiwan \\ ${ }^{3}$ Department of Public Health, China Medical University, Taiwan \\ ${ }^{4}$ Management Office for Health Data, China Medical University Hospital, Taiwan \\ ${ }^{5}$ College of Medicine, China Medical University, Taiwan \\ ${ }^{6}$ Graduate Institute of Clinical Medical Science and School of Medicine, College of Medicine, China Medical University, Taiwan \\ ${ }^{7}$ Department of Nuclear Medicine and PET Center, China Medical University Hospital, Taiwan \\ Correspondence should be addressed to Hong-Mo Shih; homoe042002@hotmail.com
}

Received 14 June 2020; Accepted 8 December 2020; Published 30 December 2020

Academic Editor: Chiara Ricci

Copyright (C) 2020 Jia-Lun Huang et al. This is an open access article distributed under the Creative Commons Attribution License, which permits unrestricted use, distribution, and reproduction in any medium, provided the original work is properly cited.

\begin{abstract}
Background. Preeclampsia is a multiple organ dysfunction during pregnancy, including hepatic, renal, and neurological dysfunction, and is defined as hypertension and proteinuria occurring after 20 weeks of pregnancy. Clinical features seen in preeclampsia are due to relatively poorly perfused placenta and maternal endothelial dysfunction. Some studies have found that preeclampsia may cause acute pancreatitis due to microvascular abnormalities and visceral ischemia. This retrospective cohort study used the Taiwanese National Health Insurance Research Databases (NHIRD) to study the relationship between preeclampsia and the risk of pancreatitis. Methods. In total, 606,538 pregnant women were selected from the NHIRD between January 1, 1998 and December 31, 2010. They were divided into a preeclampsia cohort $(n=485,211)$ and a nonpreeclampsia cohort $(n=121,327)$. After adjusting for comorbidities that may induce pancreatitis, we analyzed and compared the incidence of pancreatitis in the two cohorts. Results. The overall incidence of pancreatitis in the preeclampsia cohort was significantly higher than that in the control cohort (4.29 vs. 2.33 per 10,000 person-years). The adjusted HR of developing pancreatitis increased 1.68-fold (95\% CI: 1.19-2.36) in the preeclampsia cohort. In addition, pregnant women with preeclampsia without comorbidities had a significantly high risk of pancreatitis $(\mathrm{aHR}=1.83,95 \%$ CI 1.27-2.63). The combined effect of preeclampsia and alcoholrelated diseases resulted in the highest risk of pancreatitis $(\mathrm{aHR}=43.4,95 \% \mathrm{CI}$ : 6.06-311.3). Conclusion. Compared with patients without preeclampsia, the risk of pancreatitis in patients with preeclampsia is significantly increased after adjusting for demographics and comorbidities. The risk of pancreatitis is greatly increased when preeclampsia is accompanied by alcoholrelated diseases, hepatitis C, gallstones, diabetes, or age of 26-35 years. Early identification and effective control of preeclampsia and the associated comorbidities can reduce the risk of pancreatitis and the associated morbidity and mortality.
\end{abstract}

\section{Introduction}

Preeclampsia is defined as the presence of de novo hypertension (>140 $\mathrm{mmHg}$ systolic or $>90 \mathrm{mmHg}$ diastolic) after 20 weeks of gestation combined with proteinuria or other maternal organ dysfunction (renal, hepatic, and neurologic diseases) [1-3]. It occurs in 3\%-5\% of pregnancies worldwide [4]. Risk factors associated with preeclampsia include a past history of preeclampsia, preexisting hypertension, preexisting diabetes, obesity, multifetal pregnancy, chronic kidney disease, advanced maternal age, antiphospholipid syndrome, and systemic lupus erythematosus [5].

The increased systemic inflammatory response plays a critical role in the pathogenesis of preeclampsia, leading to edema, extravasation, and increased damage to the vascular bed of the placenta, kidneys, and other organs [6]. This leads 
to poorly perfused placenta and maternal endothelial dysfunction [7]. These changes make preeclampsia a multiorgan syndrome dysfunction, with increased risks of various disorders, including chronic hypertension, diabetes mellitus, ischemic heart disease, cerebrovascular disease, kidney disease, thromboembolism, hypothyroidism, and even memory impairment [8]. Women with preeclampsia have an increased risk of life-threatening obstetric or medical complications. Globally, $10 \%-15 \%$ of maternal deaths due to pregnancy complications are directly related to preeclampsia/eclampsia [9].

Pancreatitis is the inflammation of the pancreatic glandular parenchyma, usually accompanied by abdominal pain and elevated serum pancreatic enzymes. Acute pancreatitis is the most common cause of hospitalization for gastrointestinal diseases in the United States [10]. Several conditions are related to acute pancreatitis, among which gallstones and chronic alcoholism account for approximately two thirds [11]. Systemic inflammatory response syndrome and organ failure are the main causes of death in the first two weeks of acute pancreatitis, and sepsis is the main cause of death after two weeks [12]. In a systematic review of studies of acute pancreatitis, the overall mortality rate is approximately $5 \%$ [13].

Severe preeclampsia can cause various systemic abnormalities, such as refractory abdominal pain, impaired liver function, severe hypertension, cerebral or visual disturbances, progressive renal insufficiency, and thrombocytopenia $[14,15]$. Some case reports have indicated a link between the development of pancreatic diseases and preeclampsia [16-20], but studies with a higher level of evidence examining this link are lacking. Therefore, this retrospective cohort study explored the relationship between preeclampsia and pancreatitis by using the Taiwanese National Health Insurance Research Database (NHIRD).

\section{Materials and Methods}

2.1. Data Source. This retrospective population-based study used data from the Taiwan NHIRD which covers medical benefit claims for over 23 million people (approximately 99\% of Taiwan's population) [21]. The NHIRD contains the registry of beneficiaries and all medical service data. These claim files record the disease based on the International Classification of Diseases, Ninth Revision, Clinical Modification (ICD-9-CM). For each beneficiary, a unique encrypted identification number is used to link all insurance information and health care records. This study was approved by the Ethics Review Board of China Medical University, Taichung (CMUH104-REC2-115).

2.2. Variables and Participants. We identified all preeclampsia patients from the NHIRD corresponding to the International Classification of Disease, the Ninth Revision (ICD-9) codes 642.4-642.7 from 1998 to 2010. The date of preeclampsia diagnosis was assigned as the index date. For each woman with preeclampsia, we identified a pregnant woman without the history of preeclampsia for the comparison cohort, frequency matched by age (every 5 years span), and pregnancy year. Patients were excluded if they were $<18$ or $>45$ years of age and had a history of pancreatitis (ICD-9 code 577) before the index date. The confirmation of pancreatitis events was based on the database from 1998 to 2011 as the study endpoint. All study participants were followed up from the index date to the occurrence of endpoint, death, withdrawal from the database, or the end of 2011, whichever came first. We evaluated several comorbidities, which were defined as the history before the endpoint. They could be related to pancreatitis, namely, alcohol-related diseases (ICD-9 codes 291, 303, 305.00, 305.01, 305.02, 305.03, 790.3, and V11.3), biliary stone (ICD-9 code 574), diabetes mellitus (ICD-9 code 250), hyperlipidemia (ICD-9 code 272), hypertension (ICD-9 codes 401-405), hepatitis B virus (ICD-9 codes V02.61, $070.20,070.22,070.30$, and 070.32), and hepatitis $C$ virus (ICD-9 codes V02.62, 070.41, 070.44, 070.51, and 070.54).

2.3. Statistical Analysis. Distributions of age and comorbidities were compared between cohorts with and without preeclampsia and then tested using the chi-square test for categorical variables and Student's $t$-test for continuous variables. The cumulative incidence curve for pancreatitis was assessed using the Kaplan-Meier method, and intergroup differences were estimated using the log-rank test. We estimated the incidence densities of pancreatitis during followup in both cohorts. We evaluated the risk of pancreatitis for preeclampsia patients compared with the comparison cohort by using univariable and multivariable Cox proportional hazards models and presented by hazard ratios (HRs) and corresponding 95\% confidence intervals (CIs). Multivariable models were adjusted for age and comorbidities. All statistical analyses were performed using SAS 9.4 (SAS Institute, Cary, NC, USA). We set the significant level at $p<0.05$ for two-sided testing.

\section{Results}

The eligible study participants consisted of 17263 patients in the preeclampsia cohort and 69052 individuals in the nonpreeclampsia cohort. The baseline characteristics of all patients are summarized in Table 1 . The mean age of patients with and without preeclampsia was $31.2 \pm 5.19$ and $31.0 \pm$ 5.22 years, respectively, and $64.0 \%$ of the study participants were aged $26-35$ years. Compared with patients without preeclampsia, those with preeclampsia were more likely to have biliary stones (1.74\%), diabetes mellitus (5.02\%), hyperlipidemia (1.81\%), hypertension (3.85\%), hepatitis B virus (0.74\%), and hepatitis $\mathrm{C}$ virus $(0.27 \%)$. The average followup duration was $7.03 \pm 3.90$ years for the preeclampsia cohort and $7.03 \pm 3.92$ years for the comparison cohort.

The overall incidence of pancreatitis was greater in the preeclampsia cohort than in the comparison cohort $(4.29$ vs. 2.33 per 10,000 person-years, crude $\mathrm{HR}=1.84,95 \% \mathrm{CI}$ 1.32-2.55), and after adjusting for age and comorbidities of alcohol-related disease, biliary stone, diabetes mellitus, hyperlipidemia, hypertension, hepatitis B virus, and hepatitis $\mathrm{C}$ virus, the adjusted HR (aHR) was 1.68 (95\% CI 1.55-1.78) (Table 2). The pancreatitis incidence increased with age. The risk of pancreatitis was higher in patients with preeclampsia 
TABLE 1: Demographic characteristics and comorbidities in patients with and without preeclampsia.

\begin{tabular}{lccc}
\hline \multirow{2}{*}{ Characteristics } & \multicolumn{2}{c}{ Preeclampsia } & \\
& $(N=69052)$ & $(N=17263)$ & \\
\hline Age stratified & & & 0.99 \\
$18-25$ & $8544(12.4)$ & $2136(12.4)$ & \\
$26-35$ & $44216(64.0)$ & $11054(64.0)$ & \\
$36-45$ & $16292(23.6)$ & $4073(23.6)$ & \\
Age, mean $\pm S D^{\dagger}$ & $31.0 \pm 5.22$ & $31.2 \pm 5.19$ & 0.01 \\
Comorbidity & & & \\
Alcohol-related disease & $64(0.09)$ & $16(0.09)$ & 0.99 \\
Biliary stone & $722(1.05)$ & $300(1.74)$ & $<0.001$ \\
Diabetes mellitus & $358(0.52)$ & $866(5.02)$ & $<0.001$ \\
Hyperlipidemia & $236(0.34)$ & $312(1.81)$ & $<0.001$ \\
Hypertension & $293(0.42)$ & $664(3.85)$ & $<0.001$ \\
Hepatitis B & $385(0.56)$ & $127(0.74)$ & 0.006 \\
Hepatitis C & $103(0.15)$ & $46(0.27)$ & $<0.001$ \\
\hline
\end{tabular}

Chi-square test, ${ }^{\dagger} t$-test.

than the comparison cohort for the 26-35 age group $(\mathrm{aHR}=2.22,95 \%$ CI 1.43-3.44). Moreover, among patients without comorbidities, the risk of pancreatitis was higher in the preeclampsia cohort than in the comparison cohort $(\mathrm{aHR}=1.83,95 \%$ CI 1.27-2.63). Patients with preeclampsia had a higher pancreatitis rate than did the comparison cohort after 14 years of follow-up (log-rank $p<0.001$, Figure 1$)$.

Table 3 reveals the combined effect of preeclampsia with specific comorbidities. Compared with nonpreeclampsia patients without alcohol-related diseases, a significantly increased risk of pancreatitis was observed in preeclampsia patients with alcohol-related diseases $(\mathrm{aHR}=43.4,95 \% \mathrm{CI}$ 6.06-311.3), followed by preeclampsia patients without alcohol-related diseases (aHR $=1.80,95 \%$ CI 1.29-2.50) (Table 3). A significantly high pancreatitis risk was observed in patients with both preeclampsia and hepatitis $\mathrm{C}$ virus $(\mathrm{aHR}=11.0,95 \%$ CI 1.54-78.9) compared with those without either disease. Preeclampsia coexisting with diabetes mellitus $(\mathrm{aHR}=5.89,95 \%$ CI $1.87-18.6)$ or biliary stone $(\mathrm{aHR}=3.63$, 95\% CI 1.59-8.27) was also associated with a high risk of pancreatitis.

\section{Discussion}

This is the first nationwide, population-based study to investigate the incidence of pancreatitis after preeclampsia. Our results revealed that patients with a history of preeclampsia exhibited a 1.68-fold risk of pancreatitis than did those without preeclampsia, after adjusting for demographic characteristics and comorbidities.

Preeclampsia is a common pregnancy-specific disease with potential adverse maternal and neonatal outcomes affecting $3 \%-5 \%$ of all pregnancies [4]. Preeclampsia is an obstetric emergency; in the United States, preeclampsia/eclampsia is one of the four leading causes of death among pregnant women, along with bleeding, cardiovascular dis- ease, and thromboembolism [22-24]. Women with preeclampsia are more likely to suffer from systemic diseases in the future, including hypertension, coronary heart disease, stroke, diabetes, and renal disease $[25,26]$. Our study found that women with preeclampsia are more likely to develop comorbidities such as gallstones, diabetes, hyperlipidemia, hypertension, and hepatitis B and C infection than pregnant women without preeclampsia (Table 1), which is consistent with some existing studies [27-30]. In fact, these comorbidities are also risk factors for pancreatitis [31-34]. Pancreatitis may affect surrounding tissues or may cause dysfunction of the distal organ system. A small proportion of patients may have pancreatic necrosis, inflammation of surrounding tissues, and organ failure $[35,36]$. The pathophysiology of pancreatitis has not been fully elucidated $[37,38]$. The most common factors associated with acute pancreatitis are gallstones (35\%-75\%) and alcohol consumption (25\%-35\%), followed by idiopathic causes (10\%-20\%), hypertriglyceridemia $(1 \%-4 \%)$, endoscopic retrograde cholangiopancreatography, and drugs $(1.4 \%-2 \%) \quad[31-34,39]$. Preeclampsia and pancreatitis seem to have common risk factors, including diabetes, hyperlipidemia, and hepatitis $[8$, 29-34, 39, 40].

The overall incidence of pancreatitis in the preeclampsia cohort was higher than that in the control cohort, even after adjusting for comorbidities associated with pancreatitis and even when only preeclampsia patients without comorbidities were considered (Table 2). Compared with the control cohort, the incidence of pancreatitis in patients with preeclampsia increased with age, especially in the 26-35 age group; this finding is consistent with studies indicating that advanced maternal age is prone to preeclampsia or pancreatitis $[41,42]$.

The present study revealed that preeclampsia was an independent risk factor for pancreatitis. However, specific diseases, such as alcohol-related diseases, gallstones, or diabetes, were more influential than preeclampsia. Preeclampsia patients with a single specific comorbidity had a higher risk of pancreatitis than did nonpreeclampsia patients with that comorbidity (aHRs $=1.78-1.89)$ (Table 3 ), but the combined effects of preeclampsia and the specific comorbidity further increased the risk of pancreatitis. The risk was especially high for preeclampsia combined with alcohol-related diseases, as well as with hepatitis $\mathrm{C}$, diabetes mellitus, and biliary stone.

The prevalence of pancreatitis in pregnancy is low and ranges between 1 in 1000 and 1 in 3000 deliveries [43], but maternal mortality with severe pancreatitis is high [44]. The incidence of fetal distress and fetal loss increases with the severity of pancreatitis [45]. Fortunately, early diagnosis of pancreatitis during pregnancy and improved maternal-infant intensive care has led to a declined in maternal and infant mortality rates [44]. The most common causes of acute pancreatitis in pregnancy are gallstones (67\%-100\% of pregnancy cases) [43], alcoholism, and hypertriglyceridemia [44]. Other causes include idiopathic pancreatitis, gestational hypertension, drug-induced pancreatitis, traumatic pancreatitis, and inherited diseases $[42,44,46]$. 
TABLE 2: Incidence densities of the pancreatitis hazard ratio in women with and without preeclampsia stratified by age and presence of comorbidity.

\begin{tabular}{|c|c|c|c|c|c|c|c|c|}
\hline \multirow{3}{*}{ Variables } & \multicolumn{6}{|c|}{ Preeclampsia } & \multirow{3}{*}{ Crude HR* $(95 \%$ CI) } & \multirow{3}{*}{ Adjusted $\mathrm{HR}^{\dagger}(95 \% \mathrm{CI})$} \\
\hline & & No & & & Yes & & & \\
\hline & Event & PY & Rate $^{\#}$ & Event & PY & Rate $^{\#}$ & & \\
\hline All & 113 & 485211 & 2.33 & 52 & 121327 & 4.29 & $1.84(1.32,2.55)^{* * *}$ & $1.68(1.19,2.36)^{* *}$ \\
\hline \multicolumn{9}{|c|}{ Stratify age } \\
\hline$\leq 25$ & 19 & 66230 & 2.87 & 3 & 16945 & 1.77 & $0.61(0.18,2.08)$ & $0.58(0.17,1.99)$ \\
\hline $26-35$ & 57 & 311988 & 1.83 & 34 & 77967 & 4.36 & $2.39(1.56,3.65)^{* * *}$ & $2.22(1.43,3.44)^{* * * *}$ \\
\hline $36-45$ & 37 & 106993 & 3.46 & 15 & 26416 & 5.68 & $1.63(0.90,2.97)$ & $1.40(0.74,2.64)$ \\
\hline \multicolumn{9}{|c|}{ Comorbidity $^{\ddagger}$} \\
\hline No & 99 & 469860 & 2.11 & 41 & 106185 & 3.86 & $1.83(1.27,2.63)^{* *}$ & $1.83(1.27,2.63)^{* *}$ \\
\hline Yes & 14 & 15351 & 9.12 & 11 & 15143 & 7.26 & $0.80(0.36,1.75)$ & $0.80(0.36,1.76)$ \\
\hline
\end{tabular}

Rate $^{\#}$ : incidence rate per 10000 person-years; crude $\mathrm{HR}^{*}$ : relative hazard ratio; adjusted $\mathrm{HR}^{\dagger}$ : multivariable analysis including age, alcohol-related diseases, biliary stone, diabetes mellitus, hyperlipidemia, hypertension, hepatitis B, and hepatitis C; comorbidity ${ }^{\ddagger}$ : patients with any one of the comorbidities alcoholrelated diseases, biliary stone, diabetes mellitus, hyperlipidemia, hypertension, hepatitis $\mathrm{B}$, and hepatitis $\mathrm{C}$ were classified as the comorbidity group. ${ }^{*} p<$ $0.05,{ }^{* *} p<0.01,{ }^{* * *} p<0.001$.

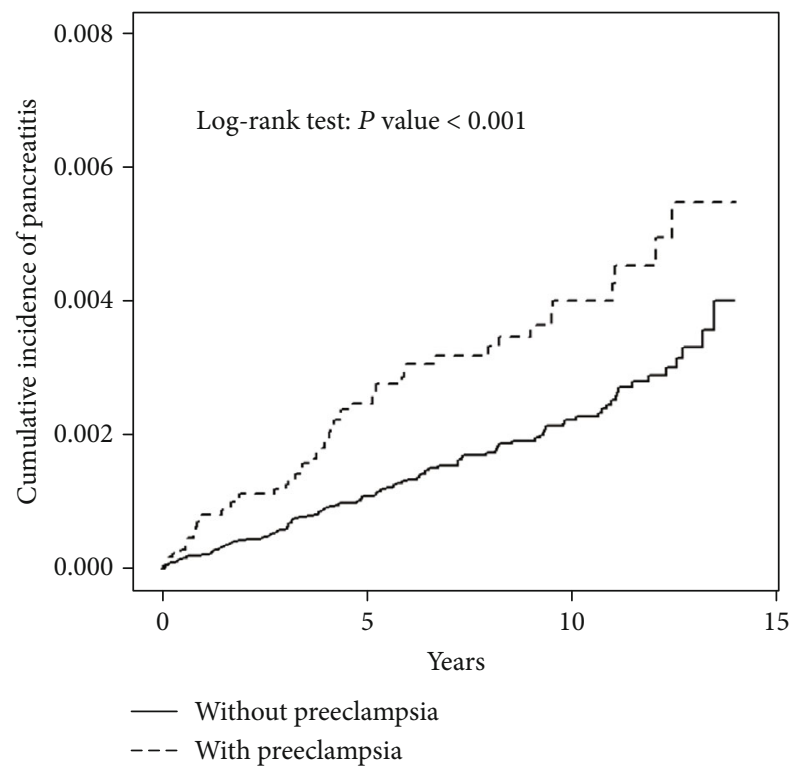

FIGURE 1: Comparison of cumulative incidence of pancreatitis among patients with preeclampsia and patients in the comparison cohort.

This study explored the relationship between preeclampsia and pancreatitis. Although no direct link was seen between preeclampsia and the pathology of pancreatitis, preeclampsia has been shown to cause global vascular endothelial dysfunction, which can lead to pancreatitis [7]. The pathophysiology of preeclampsia likely involves both maternal and placental factors, such as abnormalities in the uterine and placental circulations. The resultant ischemic placenta seems to introduce complex factors into the maternal circulation; this leads to maternal vascular endothelial dysfunction and eventually gives rise to the clinical manifestations of preeclampsia $[4,47-50]$. Preeclampsia is associated with microvascular abnormalities, which may involve the cerebral, placental, hepatic, renal, and visceral circulation. Thus, the pancreatic vasculature may also get affected and cause acute pancreatitis, which leads to organized pancreatic necrosis [20]. Ramin et al. ${ }^{43}$ investigated 9 cases of pancreatitis related to preeclampsia and found microthrombosis, intravascular coagulation, and vasculitis during preeclampsia, probably resulting in neurological, renal, hepatic, and placental diseases [43]. Another study reported that preeclampsia can cause acute edematous pancreatitis likely related to microvascular abnormalities and visceral ischemia [51]. Taken together, these findings indicate that maternal vascular endothelial dysfunction in patients with preeclampsia may increase pancreatic vascular system damage, eventually leading to pancreatitis. Further research is warranted to elucidate underlying biological mechanisms.

The difference in the cumulative incidence between patients with preeclampsia and control cohorts increased over time (Figure 1), indicating that the risk of pancreatitis in patients with a history of preeclampsia is long lasting. Because other comorbidities, such as alcoholism, gallstones, hypertension, dyslipidemia, diabetes, and overweight, further increase the risk of pancreatitis, both preeclampsia and comorbidities should be identified as soon as possible and treated effectively to reduce the patient's long-term morbidity and mortality.

\section{Limitations}

The strength of our research was its population-based design. However, the use of an observational database (i.e., NHIRD) has some inherent limitations.

First, the diagnostic accuracy was based on administrative data, making potential misjudging of preeclampsia and pancreatitis results inevitable. However, the Bureau of National Health Insurance randomly cross-checks medical records in all medical institutions to reduce error codes and misclassification bias. We identified hypertensive disorders during pregnancy and other comorbidities by using ICD-9$\mathrm{CM}$ codes. In Taiwan, since the implementation of the National Health Insurance Program, prenatal care has been very well delivered [52]. The program includes 10 routine 
TABLE 3: Cox proportional model to measure the pancreatitis hazard ratio in patients with or without comorbidities.

\begin{tabular}{|c|c|c|c|c|c|}
\hline \multicolumn{2}{|c|}{ Variables } & \multirow{2}{*}{ Event } & \multirow{2}{*}{ PY } & \multirow{2}{*}{ Rate $^{\dagger}$} & \multirow{2}{*}{$\begin{array}{c}\text { Adjusted } \mathrm{HR}^{\dagger} \\
(95 \% \mathrm{CI})\end{array}$} \\
\hline & & & & & \\
\hline - & - & 113 & 484731 & 2.33 & 1 (reference) \\
\hline - & + & 0 & 480 & 0.00 & - \\
\hline+ & - & 51 & 121225 & 4.21 & $1.80(1.29,2.50)^{* * *}$ \\
\hline+ & + & 1 & 102 & 97.7 & $43.4(6.06,311.3)^{* * *}$ \\
\hline Preeclampsia & Biliary stone & & & & \\
\hline- & - & 109 & 482191 & 2.26 & 1 (reference) \\
\hline- & + & 4 & 3020 & 13.2 & $5.45(2.01,14.8)^{* * *}$ \\
\hline+ & - & 46 & 114489 & 4.02 & $1.78(1.26,2.51)^{* *}$ \\
\hline+ & + & 6 & 6838 & 8.77 & $3.63(1.59,8.27)^{* *}$ \\
\hline Preeclampsia & Diabetes mellitus & & & & \\
\hline- & - & 104 & 479566 & 2.17 & 1(reference) \\
\hline - & + & 9 & 5645 & 15.9 & $7.13(3.61,14.1)^{* * *}$ \\
\hline+ & - & 49 & 119078 & 4.11 & $1.89(1.35,2.66)^{* * *}$ \\
\hline+ & + & 3 & 2249 & 13.3 & $5.89(1.87,18.6)^{* * *}$ \\
\hline Preeclampsia & Hyperlipidemia & & & & \\
\hline- & - & 112 & 483311 & 2.32 & 1 (reference) \\
\hline- & + & 1 & 1900 & 5.26 & $2.12(0.30,15.2)$ \\
\hline+ & - & 51 & 118648 & 4.30 & $1.85(1.33,2.57)^{* * *}$ \\
\hline+ & + & 1 & 2679 & 3.73 & $1.51(0.21,10.9)$ \\
\hline Preeclampsia & Hypertension & & & & \\
\hline- & - & 113 & 482869 & 2.34 & 1(reference) \\
\hline- & + & 0 & 2341 & 0.00 & - \\
\hline+ & - & 51 & 116693 & 4.37 & $1.86(1.34,2.59)^{* * *}$ \\
\hline+ & + & 1 & 4635 & 2.16 & $0.91(0.13,6.52)$ \\
\hline Preeclampsia & Hepatitis B virus & & & & \\
\hline- & - & 113 & 482353 & 2.34 & 1 (reference) \\
\hline - & + & 0 & 2858 & 0.00 & - \\
\hline+ & - & 51 & 120381 & 4.24 & $1.80(1.29,2.51)^{* * *}$ \\
\hline+ & + & 1 & 946 & 10.6 & $4.58(0.64,32.8)$ \\
\hline Preeclampsia & Hepatitis $\mathrm{C}$ virus & & & & \\
\hline- & - & 112 & 484455 & 2.31 & 1 (reference) \\
\hline- & + & 1 & 756 & 13.2 & $5.55(0.78,39.8)$ \\
\hline+ & - & 51 & 120965 & 4.22 & $1.82(1.30,2.53)^{* * *}$ \\
\hline+ & + & 1 & 363 & 27.6 & 11.y $(1.54,78.9)^{*}$ \\
\hline
\end{tabular}

Rate ${ }^{\dagger}$ : incidence rate per 10000 person-years; adjusted $\mathrm{HR}^{\dagger}$ : multivariable analysis including age; ${ }^{* *} p<0.01,{ }^{* * *} p<0.001$.

antenatal checkups, which involve blood pressure measurement and urinary protein tests, allowing accurate and timely diagnosis of preeclampsia by obstetricians. Although we could not calculate the validity of diagnostic codes for hypertensive disorders in pregnancy, the high validity of the diagnostic codes of the NHIRD has been reported [53, 54]. In addition, we used univariable and multivariable Cox proportional hazards models to assess the risk of pancreatitis and expressed them as HRs. Multivariable models were adjusted for age and comorbidities, including the exclusion of only hypertensive patients. The diagnosis of pancreatitis is typi- cally based on clear guidelines involving characteristic symptoms and signs, blood biochemistry results, and imaging findings [55] and is thus not prone to error.

Second, the NHIRD does not include details of high alcohol consumption, body mass index, tobacco use, or other lifestyle-related factors that may be potential confounders. We tried to reduce these confounders by, for example, including alcohol-related diseases as a proxy of alcohol consumption and metabolic syndrome parameters (such as hyperlipidemia and diabetes) as an alternative indicator of body mass index. In general, the smoking rate among 
Taiwanese women is low, being $<2.5 \%$ among pregnant women [56]. Moreover, such a large population-based study may have neutralized this effect.

\section{Conclusions}

Compared with patients without preeclampsia, the risk of pancreatitis in patients with preeclampsia was found to be significantly high even after adjusting for certain demographic characteristics and comorbidities. Preeclampsia is an independent risk factor for pancreatitis, and the risk is further increased when accompanied by alcohol-related diseases, gallstones, diabetes, hepatitis $\mathrm{C}$ infection, or age of 26-35 years. Thus, timely identification and effective management of preeclampsia and the aforementioned comorbidities can reduce the risk of pancreatitis and the associated morbidity and mortality.

\section{Abbreviations}

$\begin{array}{ll}\text { aHR: } & \text { Adjusted hazard ratio } \\ \text { CI: } & \text { Confidence interval } \\ \text { HR: } & \text { Hazard ratio } \\ \text { ICD-9-CM: } & \text { International Classification of Diseases, Ninth } \\ & \text { Revision, Clinical Modification } \\ \text { NHIRD: } & \text { National Health Insurance Research Databases. }\end{array}$

\section{Data Availability}

The dataset used in this study is held by the Taiwan Ministry of Health and Welfare (MOHW). The Ministry of Health and Welfare must approve our application to access this data. Any researcher interested in accessing this dataset can apply form to the Ministry of Health and Welfare requesting access. Please contact the staff of MOHW (email: stcarolwu@mohw.gov.tw) for further assistance: Taiwan Ministry of Health and Welfare Address: No.488, Sec. 6, Zhongxiao E. Rd., Nangang Dist., Taipei City 115, Taiwan. Phone: +886-2-8590-6848. All relevant data are within the paper.

\section{Additional Points}

Reporting Checklist. The authors have completed the STROBE reporting checklist. Data Sharing Statement. The dataset used in this study is held by the Taiwan Ministry of Health and Welfare (MOHW). The Ministry of Health and Welfare must approve our application to access this data. Any researcher interested in accessing this dataset can apply form to the Ministry of Health and Welfare requesting access. Please contact the staff of MOHW (Email: stcarolwu@mohw.gov.tw) for further assistance: Taiwan Ministry of Health and Welfare Address: No.488, Sec. 6, Zhongxiao E. Rd., Nangang Dist., Taipei City 115, Taiwan. Phone: +886-2-8590-6848. All relevant data are within the paper.

\section{Ethical Approval}

The authors are accountable for all aspects of the work in ensuring that questions related to the accuracy or integrity of any part of the work which are appropriately investigated and resolved. This study was exempted from a full ethical review by the China Medical University and Hospital Research Ethics Committee (IRB permit number: CMUH104-REC2-115-R4).

\section{Conflicts of Interest}

All authors have completed the ICMJE uniform disclosure form. The authors have no conflicts of interest to declare.

\section{Authors' Contributions}

(I) Jia-Lun Huang and Hong-Mo Shih performed the conception and design. (II) Wei-Kung Chen and Chia-Hung Kao contributed to the administrative support. (III) Jia-Lun Huang, Cheng-Li Lin, and Hong-Mo Shih contributed to the provision of study, materials, or patients. (IV) Cheng-Li Lin and Wei-Kung Chen performed the collection and assembly of data. (V) Jia-Lun Huang, Cheng-Li Lin, and Hong-Mo Shih contributed to the data analysis and interpretation. (VI) All authors contributed to the manuscript writing. (VII) All authors have approved the final manuscript.

\section{Acknowledgments}

This work was supported in part by the Taiwan Ministry of Health and Welfare Clinical Trial Center (MOHW109TDU-B-212-114004), MOST Clinical Trial Consortium for Stroke (MOST 108-2321-B-039-003-), China Medical University Hospital (DMR-109-058), and Tseng-Lien Lin Foundation, Taichung, Taiwan.

\section{References}

[1] M. E. Helewa, R. F. Burrows, J. Smith, K. Williams, P. Brain, and S. W. Rabkin, "Report of the Canadian Hypertension Society Consensus Conference: 1. Definitions, evaluation and classification of hypertensive disorders in pregnancy," CMAJ: Canadian Medical Association journal = journal de l'Association medicale canadienne, vol. 157, pp. 715-725, 1997.

[2] B. W. J. Mol, C. T. Roberts, S. Thangaratinam, L. A. Magee, C. J. M. de Groot, and G. J. Hofmeyr, "Pre-eclampsia," Lancet, vol. 387, pp. 999-1011, 2016.

[3] J. M. Pauli and J. T. Repke, "Preeclampsia: short-term and long-term implications," Obstetrics and Gynecology Clinics of North America, vol. 42, no. 2, pp. 299-313, 2015.

[4] J. M. Roberts and C. W. Redman, "Pre-eclampsia: more than pregnancy-induced hypertension," Lancet, vol. 341, no. 8858, pp. 1447-1451, 1993.

[5] K. Duckitt and D. Harrington, "Risk factors for pre-eclampsia at antenatal booking: systematic review of controlled studies," BMJ, vol. 330, p. 565, 2005.

[6] D. Mihu, C. Razvan, A. Malutan, and C. Mihaela, "Evaluation of maternal systemic inflammatory response in preeclampsia," Taiwanese Journal of Obstetrics \& Gynecology, vol. 54, no. 2, pp. 160-166, 2015.

[7] J. M. Roberts, "Endothelial dysfunction in preeclampsia," Seminars in Reproductive Endocrinology, vol. 16, no. 1, pp. 5-15, 1998.

[8] R. J. Levine, L. J. Vatten, G. L. Horowitz et al., "Pre-eclampsia, soluble fms-like tyrosine kinase 1 , and the risk of reduced 
thyroid function: nested case-control and population based study," Bmj, vol. 339, article b4336, 2009.

[9] L. Duley, "The global impact of pre-eclampsia and eclampsia," Seminars in Perinatology, vol. 33, no. 3, pp. 130-137, 2009.

[10] A. F. Peery, E. S. Dellon, J. Lund et al., "Burden of gastrointestinal disease in the United States: 2012 update," Gastroenterology, vol. 143, pp. 1179-1187, 2012.

[11] J. Toouli, M. Brooke-Smith, C. Bassi et al., "Guidelines for the management of acute pancreatitis," Journal of Gastroenterology and Hepatology, vol. 17, Suppl, pp. S15-S39, 2002.

[12] B. Gloor, C. A. Müller, M. Worni, M. E. Martignoni, W. Uhl, and M. W. Büchler, "Late mortality in patients with severe acute pancreatitis," The British Journal of Surgery, vol. 88, no. 7, pp. 975-979, 2001.

[13] P. A. Banks and M. L. Freeman, "Practice guidelines in acute pancreatitis," The American Journal of Gastroenterology, vol. 101, no. 10, pp. 2379-2400, 2006.

[14] A. C. O. G. Practice Bulletin No, "202: gestational hypertension and preeclampsia," Obstetrics and Gynecology, vol. 133, pp. e1-e25, 2019.

[15] Report of the American College of Obstetricians and Gynecologists' Task Force on Hypertension in Pregnancy, "Hypertension in pregnancy," Obstetrics and Gynecology, vol. 122, no. 5, pp. 1122-1131, 2013.

[16] C. Chan and M. Mukerji, "Non-gallstone acute pancreatitis and pre-eclampsia: a case report," Case reports in women's health, vol. 19, article e00063, 2018.

[17] S. Gainder, P. Arora, S. C. Saha, and L. Kaman, "Acute pancreatitis with eclampsia-preeclampsia syndrome and poor maternal outcome: two case reports and review of literature," Obstetric medicine, vol. 8, no. 3, pp. 146-148, 2015.

[18] M. Swank, M. Nageotte, and T. Hatfield, "Necrotizing pancreatitis associated with severe preeclampsia," Obstetrics and Gynecology, vol. 120, Part 2, pp. 453-455, 2012.

[19] M. Bahloul, M. Ayedi, H. Dammak, and K. Trabelsi, "Pancréatite aiguë nécrotico-hémorragique secondaire à une hypertension artérielle gravidique," Annales francaises d'anesthesie et de reanimation, vol. 23, no. 2, pp. 157-159, 2004.

[20] M. S. Parmar, "Pancreatic necrosis associated with preeclampsia-eclampsia," JOP: Journal of the pancreas, vol. 5, no. 2, pp. 101-104, 2004.

[21] National Health Insurance Administration and Ministry of Health and Welfare, Taiwan, R.O.C. National Health Insurance Annual Report 2017-2018, December 2020, Article in Chinese, https://www.nhi.gov.tw/english/Content_List .aspx?n=8FC0974BBFEFA56D\&topn=ED4A30E51A609E49.

[22] C. J. Berg, J. Chang, L. Elam-Evans et al., Pregnancy-related mortality surveillance-United States, 1991-1999, vol. 52, Morbidity and mortality weekly report Surveillance summaries, Washington, DC, 2003.

[23] E. K. Main, "Maternal mortality: new strategies for measurement and prevention," Current Opinion in Obstetrics \& Gynecology, vol. 22, no. 6, pp. 511-516, 2010.

[24] K. A. P. Mac, C. J. Berg, X. Liu, C. Duran, and D. L. Hoyert, "Changes in pregnancy mortality ascertainment: United States, 1999-2005," Obstetrics and Gynecology, vol. 118, pp. 104-110, 2011.

[25] L. H. Theilen, H. Meeks, A. Fraser, M. S. Esplin, K. R. Smith, and M. W. Varner, "Long-term mortality risk and life expectancy following recurrent hypertensive disease of pregnancy,"
American journal of obstetrics and gynecology, vol. 219, pp. 107.e101-107.e106, 2018.

[26] M. L. Mongraw-Chaffin, P. M. Cirillo, and B. A. Cohn, "Preeclampsia and cardiovascular disease death: prospective evidence from the child health and development studies cohort," Hypertension, vol. 56, pp. 166-171, 2010.

[27] S. Lisonkova and K. S. Joseph, "Incidence of preeclampsia: risk factors and outcomes associated with early- versus late-onset disease," American journal of obstetrics and gynecology, vol. 209, pp. 544.e512-544.e541, 2013.

[28] T. L. Weissgerber and L. M. Mudd, "Preeclampsia and diabetes," Current Diabetes Reports, vol. 15, no. 3, p. 9, 2015.

[29] I. D. Gallos, K. Sivakumar, M. D. Kilby, A. Coomarasamy, S. Thangaratinam, and M. Vatish, "Pre-eclampsia is associated with, and preceded by, hypertriglyceridaemia: a meta-analysis," BJOG, vol. 120, no. 11, pp. 1321-1332, 2013.

[30] A. Karampatou, X. Han, L. A. Kondili et al., "Premature ovarian senescence and a high miscarriage rate impair fertility in women with HCV," Journal of Hepatology, vol. 68, no. 1, pp. 33-41, 2018.

[31] A. B. Lowenfels, P. Maisonneuve, and T. Sullivan, "The changing character of acute pancreatitis: epidemiology, etiology, and prognosis," Current Gastroenterology Reports, vol. 11, no. 2, pp. 97-103, 2009.

[32] M. Al-Haddad and M. B. Wallace, "Diagnostic approach to patients with acute idiopathic and recurrent pancreatitis, what should be done?," World Journal of Gastroenterology, vol. 14, no. 7, pp. 1007-1010, 2008.

[33] D. Yadav and C. S. Pitchumoni, "Issues in hyperlipidemic pancreatitis," Journal of Clinical Gastroenterology, vol. 36, no. 1, pp. 54-62, 2003.

[34] W. M. Steinberg, S. T. Chari, C. E. Forsmark et al., "Controversies in clinical Pancreatology," Pancreas, vol. 27, no. 2, pp. 103-117, 2003.

[35] M. S. Petrov, S. Shanbhag, M. Chakraborty, A. R. Phillips, and J. A. Windsor, "Organ failure and infection of pancreatic necrosis as determinants of mortality in patients with acute pancreatitis," Gastroenterology, vol. 139, no. 3, pp. 813-820, 2010.

[36] R. Mofidi, M. D. Duff, S. J. Wigmore, K. K. Madhavan, O. J. Garden, and R. W. Parks, "Association between early systemic inflammatory response, severity of multiorgan dysfunction and death in acute pancreatitis," The British Journal of Surgery, vol. 93, no. 6, pp. 738-744, 2006.

[37] G. J. Wang, C. F. Gao, D. Wei, C. Wang, and S. Q. Ding, "Acute pancreatitis: etiology and common pathogenesis," World Journal of Gastroenterology, vol. 15, no. 12, pp. 1427-1430, 2009.

[38] R. P. Sah, R. K. Dawra, and A. K. Saluja, "New insights into the pathogenesis of pancreatitis," Current Opinion in Gastroenterology, vol. 29, no. 5, pp. 523-530, 2013.

[39] Y. K. Cheon, K. B. Cho, J. L. Watkins et al., "Frequency and severity of post-ERCP pancreatitis correlated with extent of pancreatic ductal opacification," Gastrointestinal Endoscopy, vol. 65, no. 3, pp. 385-393, 2007.

[40] D. M. Parenti, W. Steinberg, and P. Kang, "Infectious causes of acute pancreatitis," Pancreas, vol. 13, no. 4, pp. 356371, 1996.

[41] E. Bartsch, K. E. Medcalf, A. L. Park, and J. G. Ray, "Clinical risk factors for pre-eclampsia determined in early pregnancy: systematic review and meta-analysis of large cohort studies," BMJ, vol. 353, article i1753, 2016. 
[42] O. Igbinosa, S. Poddar, and C. Pitchumoni, "Pregnancy associated pancreatitis revisited," Clinics and Research in Hepatology and Gastroenterology, vol. 37, no. 2, pp. 177-181, 2013.

[43] K. D. Ramin, S. M. Ramin, S. D. Richey, and F. G. Cunningham, "Acute pancreatitis in pregnancy," American Journal of Obstetrics and Gynecology, vol. 173, no. 1, pp. 187-191, 1995.

[44] G. Ducarme, F. Maire, P. Chatel, D. Luton, and P. Hammel, "Acute pancreatitis during pregnancy: a review," Journal of Perinatology, vol. 34, no. 2, pp. 87-94, 2014.

[45] M. Tang, J. M. Xu, S. S. Song, Q. Mei, and L. J. Zhang, "What may cause fetus loss from acute pancreatitis in pregnancy: analysis of 54 cases," Medicine, vol. 97, article e9755, 2018.

[46] J. J. Eddy, M. D. Gideonsen, J. Y. Song, W. A. Grobman, and P. O'Halloran, "Pancreatitis in pregnancy," Obstetrics and Gynecology, vol. 112, no. 5, pp. 1075-1081, 2008.

[47] J. W. Meekins, R. Pijnenborg, M. Hanssens, I. R. McFadyen, and A. van Asshe, "A study of placental bed spiral arteries and trophoblast invasion in normal and severe pre-eclamptic pregnancies," British Journal of Obstetrics and Gynaecology, vol. 101, pp. 669-674, 2005.

[48] L. Myatt and R. P. Webster, "Vascular biology of preeclampsia," Journal of thrombosis and haemostasis: JTH, vol. 7, no. 3, pp. 375-384, 2009.

[49] S. E. Maynard and S. A. Karumanchi, "Angiogenic factors and preeclampsia," Seminars in Nephrology, vol. 31, no. 1, pp. 3346, 2011.

[50] C. W. Redman, G. P. Sacks, and I. L. Sargent, "Preeclampsia: an excessive maternal inflammatory response to pregnancy," American Journal of Obstetrics and Gynecology, vol. 180, no. 2, pp. 499-506, 1999.

[51] N. Badja, G. Troché, J. F. Zazzo, and D. Benhamou, "Acute pancreatitis and preeclampsia-eclampsia: a case report," American Journal of Obstetrics and Gynecology, vol. 176, no. 3, pp. 707-709, 1997.

[52] T. C. Liu and C. S. Chen, "The role of Taiwan's National Health Insurance program in influencing adequate prenatal care," The International Journal of Health Planning and Management, vol. 19, no. 2, pp. 113-130, 2004.

[53] C. L. Cheng, Y. H. Kao, S. J. Lin, C. H. Lee, and M. L. Lai, "Validation of the National Health Insurance Research Database with ischemic stroke cases in Taiwan," Pharmacoepidemiology and Drug Safety, vol. 20, no. 3, pp. 236-242, 2011.

[54] C. C. Lin, M. S. Lai, C. Y. Syu, S. C. Chang, and F. Y. Tseng, "Accuracy of diabetes diagnosis in health insurance claims data in Taiwan," Taiwan yi zhi, vol. 104, no. 3, pp. 157-163, 2005.

[55] P. A. Banks, T. L. Bollen, C. Dervenis et al., "Classification of acute pancreatitis-2012: revision of the Atlanta classification and definitions by international consensus," Gut, vol. 62, pp. 102-111, 2012.

[56] C. P. Wen, T. Y. Cheng, C. L. Lin et al., "The health benefits of smoking cessation for adult smokers and for pregnant women in Taiwan," Tobacco Control, vol. 14, suppl_1, pp. i56-i61, 2005. 